\title{
Gastrectomía vertical posterior a retiro de banda gástrica*
}

\author{
Drs. XABIER DE ARETXABALA U. ${ }^{1}$, JORGE LEÓN C. ${ }^{1}$, GONZALO WIEDMAIER T. ${ }^{1}$, \\ FERNANDO MALUENDA G. ${ }^{1}$, RICARDO ROSSI F. ${ }^{1}$, CARLOS BENAVIDES C. ${ }^{1}$ \\ 1 Unidad de Cirugía Digestiva, Clínica Alemana. \\ Santiago, Chile.
}

\begin{abstract}
Sleeve gastrectomy performed simultaneously to extraction of a gastric band. Experience in 10 patients

Background: Sleeve gastrectomy is replacing gastric banding in the management of morbid obesity. Aim: To report the results of sleeve gastrectomy performed simultaneously with the extraction of a gastric band. Material and Methods: We report ten patients aged 34 to 53 years (nine women) operated between 2008 and 2009. Results: The main indication for sleeve gastrectomy was the failure of the gastric band. No complications were recorded and patients were discharged 72 hours after the procedure. Conclusions: Sleeve gastrectomy can be carried out simultaneously with the extraction of a gastric band, without increasing operative complications.
\end{abstract}

Key words: Sleeve gastrectomy, gastric band, obesity.

\section{Resumen}

Introducción: La Gastrectomía Vertical ha reemplazado a la banda gástrica en el manejo de un importante número de pacientes portadores de obesidad. El objetivo de este trabajo es mostrar los resultados de una serie de pacientes, en los que se realizó una Gastrectomía Vertical posterior a la extracción de una banda gástrica en un mismo tiempo operatorio. Método: La serie incluye a 10 pacientes operados entre Mayo de 2008 y Noviembre de 2009. La principal indicación de la Gastrectomía Vertical fue el fracaso de la banda como tratamiento de la obesidad. Resultados: En los 10 pacientes estudiados, la gastrectomía se pudo efectuar sin mayores dificultades ni diferencias con respecto al procedimiento habitual. No existieron complicaciones post operatorias, siendo la totalidad de los pacientes dados de alta dentro de las 72 horas. Conclusión: La Gastrectomía Vertical representa una alternativa de manejo en pacientes portadores de una banda gástrica con indicación de extracción de esta, ya sea por fracaso o por síntomas asociados a su presencia. El procedimiento puede efectuarse de manera segura en el mismo acto operatorio y sus resultados son comparables a los observados cuando la técnica se efectúa en pacientes que no han sido intervenidos previamente.

Palabras clave: Gastrectomía vertical, obesidad, banda gástrica, exceso de peso.

*Recibido el 23 de Marzo de 2010 y aceptado para publicación el 29 de Abril de 2010.

Correspondencia: Dr. Xabier de Aretxabala U.

Vitacura 5951, Santiago, Chile.

E-mail: xdearetxabala@ alemana.cl 


\section{Introducción}

La banda gástrica ajustable ha sido ampliamente utilizada como tratamiento de la obesidad mórbida durante los últimos 10 años ${ }^{1,2}$. La simpleza de su instalación permitió que esta técnica ganara popularidad, sin embargo, los resultados obtenidos, y la existencia de complicaciones alejadas han hecho que en la actualidad su empleo sea cuestionado, más aun frente a la aparición de la Gastrectomía Vertical ${ }^{3}$.

La Gastrectomía Vertical fue realizada por primera vez por Hess en 1988, quien la utilizó como parte de la realización del switch pancreato duodenal; Gagner, en 1999, la realiza por laparoscopia. Esta técnica se ha transformado en la actualidad, en la intervención más empleada en nuestro medio para el manejo de la obesidad ${ }^{4,5}$. Su baja morbilidad post operatoria y sus resultados en todo comparables con el by pass gástrico, en lo que dice relación al exceso de peso perdido, han determinado la alta frecuencia de su indicación ${ }^{6-9}$.

Frente a pacientes en los que se detecta el fracaso de la banda gástrica como tratamiento de la obesidad, o en los que existen complicaciones derivadas de su uso, la extracción de la banda y la realización inmediata de una Gastrectomía Vertical constituye una alternativa.

El objetivo de este trabajo es reportar una serie de pacientes que fueron sometidos a la extracción de la banda y a la realización de una Gastrectomía Vertical en el mismo tiempo operatorio.

\section{Material y Método}

La serie está compuesta por 10 pacientes quienes fueron intervenidos durante el período comprendido entre Mayo de 2008 y Noviembre de 2009 en la Clínica Alemana de Santiago.

De los pacientes, 9 fueron de sexo femenino, la edad fluctuó entre 34 y 53 años (promedio 42 años). Del total, en 4 la banda había sido instalada en la Clínica Alemana.

Previo a indicar la cirugía, todos los pacientes fueron evaluados por un comité multidisciplinario conformado por cirujanos, psiquiatras y nutriólogos.

Como parte de la evaluación preoperatoria, los pacientes fueron sometidos a una endoscopia digestiva alta y a un estudio contrastado de esófago y estómago. En todos los casos la banda fue desinflada al menos 2 semanas previo a la cirugía.

Los pacientes fueron intervenidos mediante técnica francesa y posición de Fowler. Todos recibieron aplicación de medias compresivas neumáticas durante la intervención y de heparina de bajo peso molecular desde el momento de la cirugía y hasta los 5 días desde su alta.
Para la realización del neumoperitoneo se empleó una aguja de Verres supra umbilical, el primer trocar se instaló mediante el uso de Optiview ${ }^{\circledR}$ (Ethicon Endosurgery Inc, Cincinnatti USA). El neumoperitoneo se mantuvo a $15 \mathrm{~mm}$ de $\mathrm{Hg}$.

En ambos hipocondrios se instalaron trocares accesorios de $12 \mathrm{~mm}$, mientras que un trocar de 5 $\mathrm{mm}$ se instaló en flanco izquierdo. Como método retractor del hígado, empleamos una pinza con una gasa en su extremo, instalado en epigastrio.

Posterior a la exploración abdominal, la primera maniobra a efectuar es la liberación de la cápsula fibrosa que rodea a la banda gástrica. Luego de liberadas las adherencias existentes entre el lóbulo izquierdo del hígado y la zona del dispositivo, el área de la banda es identificada. La cápsula es abierta mediante material cortante hasta liberar por completo la zona del broche (Figura 1). Seguidamente, procedemos a abrir el balón y a la sección de la banda. Luego de liberar por completo la zona de mayor grosor, la que corresponde al área del broche, mediante delicados movimientos la banda es extraída.

Extraída la banda, procedemos a la realización de la esqueletización gástrica, la que se efectúa de manera indistinta mediante el uso de bisturí harmónico (Ultrasicion; Ethicon Endosurgery, Cincinnati, OH, USA) o de un coagulador de impedancia (Ligasure, Covidien Mansfield, MA USA). Esta decisión depende exclusivamente de la preferencia del cirujano. Especial precaución debe tenerse a nivel de la zona del ángulo de His, lugar donde se encontraba la banda gástrica, debido a la existencia de adherencias a ese nivel.

Liberado el estómago, se introduce una sonda 36 French hasta la primera porción del duodeno y se realiza la sección gástrica mediante stappler desde la región antral hasta el ángulo de His. Usamos

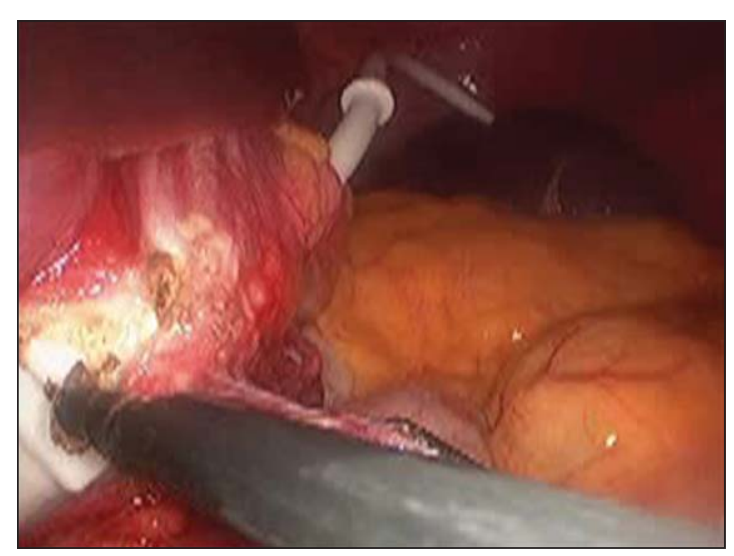

Figura 1. Apertura de la cápsula que envuelve a la banda. 
exclusivamente carga verde, dado que la pared gástrica, con mucha frecuencia, posee un grosor mayor que el habitual, como consecuencia de la existencia previa de la banda. De igual modo, especial cuidado se debe tener en no acercar demasiado la línea de sección hacia la curva menor en la región cardial. Esto con el objeto de evitar la zona del estómago donde éste se encontraba plicado sobre la banda.

La indicación de efectuar una sutura de refuerzo sobre la línea de sutura, será decisión del cirujano, no realizándose de manera constante.

Posterior a la sección gástrica, efectuamos una prueba de azul de metileno para lo cual la sonda de calibración es retirada hasta el tercio proximal del estómago, introduciendo una cantidad aproximada de 60 cc de azul de metileno, hasta observar la distensión del remanente gástrico. En todos los pacientes se deja un drenaje de Jackson-Pratt a lo largo del borde de sección. El espécimen es retirado a través del orificio del trocar ubicado en hipocondrio izquierdo. Seguidamente, efectuamos la extracción del reservorio efectuando una incisión sobre este.

Previo a realimentar al paciente vía oral, efectuamos un estudio radiológico con bario diluido, el que tiene por finalidad evaluar la ausencia de filtración y el buen vaciamiento gástrico (Figura 2).

El paciente permanece hospitalizado durante tres días, siendo dado de alta con indicaciones precisas de alimentación, empleo de bloqueadores de la bomba de protones, anticoagulación y aporte vitamínico.

Durante el post-operatorio alejado, los pacientes son controlados por miembros del equipo de obesidad de nuestro grupo de trabajo.

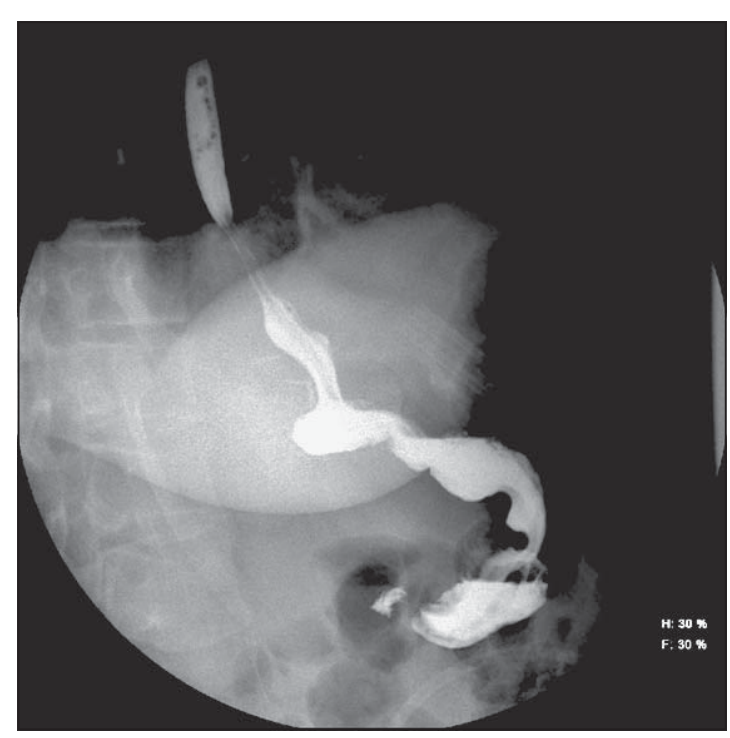

Figura 2. Imagen del estómago remanente durante el estudio contrastado.

\section{Resultados}

La indicación por la que se retiró la banda gástrica se muestra en la Tabla 1, destacando el alto número en los que el motivo fue el fracaso de la banda como método de control de la obesidad, hecho observado en la mitad de los pacientes; la existencia de sintomatología atribuida a la banda, principalmente reflujo, fue la indicación en 2 , mientras que otras dos pacientes ocurrió el deslizamiento de la banda. Finalmente, en un paciente se objetivó la desconexión entre la banda y reservorio además del fracaso del manejo de su obesidad.

La mayoría de los pacientes permaneció con la banda, durante un período superior a 2 años, en un caso, permaneció 1 año 34 días, la indicación para el retiro fue el fracaso de tratamiento (Tabla 2).

Ninguno de los pacientes presentó complicaciones durante su post operatorio, siendo la totalidad dados de alta al tercer día de la cirugía, luego del control radiológico y el inicio de la realimentación.

El IMC de los paciente, previo a la realización de la extracción de la banda y la gastrectomía, fluctuó entre 29 y $38 \mathrm{~kg} / \mathrm{m}^{2}$ (promedio: $34 \mathrm{k} / \mathrm{m}^{2}$ ). Las dos pacientes que presentaron IMC menor a 30 previo a la cirugía, fueron aquellas en las que el deslizamiento de la banda fue la indicación de la cirugía.

Transcurridos al menos 2 meses desde la operación, la totalidad de los pacientes han podido completar su esquema de realimentación, no difiriendo en nada del esquema utilizado en pacientes sometidos a gastrectomía vertical como primer procedimiento.

Tabla 1. Causas de retiro de la banda

\begin{tabular}{ll}
\hline Causa & n \\
\hline Fracaso & 5 \\
Deslizamiento & 2 \\
Esofagitis & 2 \\
Desconexión & 1 \\
\hline
\end{tabular}

Tabla 2. Tiempo con banda previo a cirugía

\begin{tabular}{lc}
\hline Tiempo con banda & n de pacientes \\
\hline$<1$ año & 0 \\
$1-2$ años & 3 \\
$2-4$ años & 5 \\
$>4$ años & 2 \\
\hline
\end{tabular}




\section{Discusión}

La banda gástrica laparoscópica fue empleada muy comúnmente en el manejo de la obesidad durante un largo período de tiempo. La principal ventaja del método radicaba en la simplicidad de su instalación ${ }^{1,2}$. Sin embargo, el seguimiento alejado de estos pacientes dejó en evidencia la existencia de dos problemas: Por una parte, los resultados en cuanto a pérdida del exceso del peso no eran del todo satisfactorios y por otro las complicaciones no sólo ocurrían durante el período post operatorio inmediato sino también ocurrían durante el post operatorio alejado, incluso años luego de la cirugía. La tasa anual de reoperación por complicaciones de la banda gástrica, fluctúa entre un 4 y un $9 \%^{10-12}$. Este hecho determina la necesidad de realizar la extracción de la banda y en muchos casos emplear un nuevo método para el tratamiento de la obesidad. La realización de un by pass gástrico posterior al retiro de una banda gástrica representa una opción válida, Spivak publica una serie de 33 pacientes en los cuales debió efectuar un by pass gástrico posterior al retiro, obteniendo buenos resultados no sólo en cuanto al exceso de peso perdido, sino también en cuanto a complicaciones post operatorias ${ }^{12}$.

Debido a que la Gastrectomía Vertical se emplea cada vez con más frecuencia para el tratamiento de la obesidad, la ejecución de dicho procedimiento posterior al retiro de la banda constituye una alternativa válida. En la actualidad, existen diversas publicaciones que muestran la factibilidad de realizar ambos procedimientos en un tiempo, como también los resultados obtenidos. Una de las mayores controversias relacionadas a la indicación de la Gastrectomía Vertical, tiene que ver con la ejecución de un procedimiento restrictivo en un paciente en el cual fracasó otro procedimiento restrictivo. Sin embargo, aunque dicha aseveración es correcta desde un punto de vista teórico, debemos considerar que una de las causas más comunes de la falla de tratamiento en la banda gástrica tiene que ver con el grado de adhesión del paciente al tratamiento, hecho que todos hemos experimentado con pacientes que no concurren a control para nuevos ajustes de sus bandas. Además, es necesario considerar, que en la Gastrectomía Vertical no sólo actúan medidas de tipo restrictivas sino también tienen importancia alteraciones de tipo hormonal que favorecerían la baja de peso ${ }^{6,89}$. Por todo lo anterior creemos que la Gastrectomía Vertical puede considerarse una opción válida en estos pacientes.

La realización de una Gastrectomía Vertical en un paciente portador de una banda gástrica, representa un mayor desafío para el cirujano que la ejecución del mismo procedimiento en un caso sin dicha ban- da. La existencia de tejido fibroso a nivel del lecho del dispositivo y las posibles alteraciones anatómicas a nivel del tercio superior del estómago le otorgan mayor dificultad al procedimiento. Debido a lo anterior existe prácticamente consenso del empleo de grapas de mayor longitud (verdes) con el objeto de lograr un cierre confiable en un tejido posiblemente engrosado, en el cual el empleo de grapas tradicionales (azules) pudiesen ser insuficientes. Otro hecho de importancia es la necesidad de evitar realizar la sección gástrica en el área de envoltura de la banda por el estómago. Si seccionamos el estómago a este nivel, lo estaremos haciendo en una zona en la que encontraremos parte de la plicatura gástrica, con la consiguiente producción de un área isquémica a ese nivel. Por este motivo y si no estamos seguros de haber logrado deshacer la envoltura gástrica sobre la banda, tratamos de alejarnos de la curva menor al momento de efectuar la sección gástrica a nivel cardial. Otro punto de importancia en el procedimiento, tiene que ver con el manejo del fondo gástrico. A diferencia de la ejecución de una manga gástrica en un paciente sin una banda previa, en estos casos existen abundantes adherencias a nivel del ángulo de His, que fijan el fondo gástrico a las estructuras retroperitoneales, haciendo más difícil su liberación. Este punto de la cirugía es clave en el éxito de la misma, debido a que de no lograr su completa liberación estaremos dejando una amplia porción de fondo gástrico remanente.

En relación a la indicación de la Gastrectomía Vertical en pacientes portadores de una banda gástrica, es nuestra conducta actual indicarla de manera rutinaria si es necesario mantener un método de control de la obesidad.

La endoscopia digestiva realizada como examen pre-operatorio tiene por objetivo, además de examinar la mucosa gástrica, descartar la posibilidad de migración de la banda hacia el interior del estómago lo que contraindicaría, a nuestro juicio, la realización del procedimiento combinado.

Los resultados obtenidos con nuestra serie, al menos en lo relacionado a complicaciones post operatorias, son auspiciosos, no diferenciándose de los pacientes sometidos a Gastrectomía Vertical como primera cirugía.

La posibilidad de esperar un intervalo de tiempo entre la extracción de la banda y la realización de la gastrectomía, aun cuando parece lógico para así permitir la disminución de la reacción inflamatoria local previo a efectuar la resección, no posee el respaldo de series publicadas demostrando dicha ventaja. El juicio clínico del cirujano al evaluar la zona a intervenir, es posiblemente la mejor manera de decidir la conveniencia o no de realizar ambos procedimientos. 
En relación a los resultados de esta serie sobre el exceso de masa corporal perdida, nuestro seguimiento es aún escaso como para analizarlo. Las series publicadas por otros grupos quirúrgicos muestran grupos de entre 8 y 25 pacientes, con pérdidas del exceso de peso de alrededor de un $50 \%{ }^{12-14}$. La adecuada selección de los pacientes para ser intervenidos representa un punto clave a considerar, más aún teniendo presente que la principal indicación de la cirugía fue el fracaso a un método quirúrgico de tratamiento.

En conclusión, la realización de una Gastrectomía Vertical posterior a la extracción de una banda gástrica en forma contemporánea, representa una alternativa factible para aquellos pacientes portadores de una banda en los que es necesario su retiro, sea este por una complicación asociada a su empleo o por falla del control de la obesidad.

\section{Referencias}

1. Chapman AE, Kiroff G, Game P, Foster B, O'Brien P, Ham J, et al. Laparoscopic adjustable gastric banding in the treatment of obesity: A systematic literature review. Surgery 2004; 135: 326-351.

2. Weiner R, Blanco-Engert R, Weiner S, Matkowitz R, Schaefer L, Pomhoff I, et al. Outcome after laparoscopic adjustable gastric banding 8 years experience Obes Surg 2003; 13: 427-434.

3. Suter M, Calmes JM, Paroz A, Giusti V. A 10 year experience with laparoscopic gastric banding for morbid obesity: High long term complication and failures rates. Obes Surg 2006; 16: 829-835.

4. Hess DS, Hess DW, Oakley RJ. Biliopancreatic diversion with duodenal switch. Obes Surg 1998; 8: 267 282.
5. Ren CJ, Patterson E, Gagner M. Early results of laparoscopic biliopancreatic diversión with duodenal switch: a case series of 40 consecutive patients. Obes Surg 2000; 10: 514-523.

6. Gumbs AA, Gagner M, Dakin G, Pomp A. Sleeve gastrectomy for morbid obesity. Obes Surg 2007; 17: 962-969.

7. Roa PE, Kaider-Person O, Pinto D,Cho M, Rosenthal RJ. Laparoscopic Sleeve gastrectomy as treatment for morbid obesity: technique and short term outcome Obes Surg 2006; 16: 1323-1326.

8. Deitel M, Crosby R, Gagner M. The first international consensus summit for sleeve gastectomy. Obes Surg 2008; 18: 487-496.

9. Baltasar A, Serra C, Pérez N, Bou R, Bengochea M, Ferri L. Laparoscopic sleeve gastrectomy: A multy purpose bariatric operation. Obesity Surgery 2005; 15: 1124-1128.

10. De Waele B, Lauwers M, Van Nieuwenhove Y, Delvaux G. Outpatient laparoscopic gastric banding: initial experience. Obes Surg 2004; 14: 1108-1110.

11. Biertho L, Steffen R, Branson R, Potoczna T, Piec G, Horber FF. Management of failed adjustable gastric banding. Surgery 2005; 137: 33-41.

12. Spivak H, Beltran OR, Slavchev P, Wilson EB. Laparoscopic revision from LAP-BAND to gastric by pass. Surg Endosc 2007; 21: 1388-1392.

13. Bernante P, Foletto M, Busetto L. Pomerri F, Vecchiato G, Prevedello L, et al. Feasibility of laparoscopic sleeve gastrectomy as a revision procedure for prior laparoscopic gastric banding. Obes Surg 2006; 16: 1327-1330.

14. Acholonu E, McBean E, Court I, Bellorin O, Szomstein S, Rosenthal RJ. Safety and short term outcomes of laparoscopic sleeve gastrectomy as a revisional approach for failed laparoscopic adjustable gastric banding in the treatment of morbid obesity. Obes Surg 2006; 16: 1327 1330 . 